\section{Análise da lógica de intervenção do Programa Nacional de Controle da Dengue}

\section{Intervention logic analysis of the National Dengue Fever Control Program}

Ana Cláudia Figueiró 1

Ana Paula Sóter 2

Cynthia Braga 3

Zulmira Maria de Araújo Hartz 4

Isabella Samico 5

\begin{abstract}
Objectives: to evaluate the logical coherence of the Brazilian National Dengue Fever Control Program (PNCD) with regard to its objectives and the measures taken to achieve results.

Methods: an evaluative study, an intervention logic analysis, using a review of documentation from three information sources on the PNCD: the report produced by a panel of specialists meeting at an international seminar to evaluate the program; technical documents and the opinions of specialists; and scientific studies. The analysis of the program was compared to the Pan-American Health Organization model for the prevention and control of dengue fever the Integrated Management Strategy-Dengue Fever.

Results: convergence was found between the strategies with regard to presuppositions, objectives, overarching goals and components, and the content of interventions. PNCD implementation was found to be weak in terms of integration at municipal level, especially in its management structure, as well as in the components analyzed, with a greater emphasis on the vector control component.

Conclusions: the model lacks coherence and there is a need to update it at different PNCD management levels, in view of the fact that little progress has been made in meeting the overall and specific objectives of the components.
\end{abstract}

Key words Dengue, National health programs, Programs evaluation
1,2,5 Diretoria de Pesquisa. Instituto de Medicina Integral Prof. Fernando Figueira. Rua dos Coelhos 300. Boa Vista. Recife, PE, Brasil. CEP: 50.070-550. E-mail: anafigueiro@imip.org.br 3 Centro de Pesquisa Aggeu Magalhães. Recife, PE, Brasil. 4 Universidade Nova de Lisboa. Lisboa, Portugal.

\section{Resumo}

Objetivos: avaliar a coerência lógica do Programa Nacional de Controle da Dengue no Brasil (PNCD) quanto aos objetivos e meios definidos para o alcance dos resultados.

Métodos: pesquisa avaliativa, análise lógica da intervenção, utilizando-se revisão documental de três fontes de informação sobre o PNCD: relatório de painel de especialistas reunidos em seminário internacional para avaliação do programa; documentos técnicos e opinião de especialistas; e estudos científicos. Comparou-se a análise do programa ao modelo da Organização Panamericana da Saúde para prevenção e controle da dengue - Estratégia de Gestão Integrada-Dengue (EGI).

Resultados: encontrou-se convergência entre as estratégias quanto aos pressupostos, objetivos, metas gerais e dos componentes, e conteúdos das intervenções. Verificou-se fragilidade na implementação do PNCD no âmbito municipal em sua integralidade, especialmente de estrutura e gestão, bem como para os componentes analisados, com maior comprometimento no componente de controle vetorial.

Conclusões: baixa coerência e necessidade de atualização do modelo teórico-lógico nos diferentes niveis de gestão do PNCD frente ao pequeno alcance dos objetivos gerais e específicos dos componentes.

Palavras-chave Dengue, Programas nacionais de saúde, Avaliação de programas 


\section{Introdução}

A dengue tem constituído um grave problema de saúde pública no mundo e a incidência de dengue nas Américas, incluindo o Brasil, apresenta tendência ascendente nas duas últimas décadas, a despeito dos inúmeros programas de erradicação ou controle implantados.1,2 Desde a década de 1980 o Brasil vem promovendo estratégias diferenciadas de intervenção para redução da ocorrência de epidemias e da morbi-mortalidade por dengue.

A estratégia atual de intervenção, desenvolvida desde 2002 por meio do Programa Nacional de Controle da Dengue (PNCD) orienta-se a partir de três fundamentos: a) construção do programa em caráter permanente; b) respeito aos princípios do Sistema Único de Saúde (SUS), com a gestão e execução do programa realizada pelos municípios e integração com as ações básicas de saúde; e c) gestão integrada dos vários componentes do programa. 3 Partindo desses pressupostos, o PNCD foi elaborado em dez componentes, e estabeleceramse como metas: redução dos índices de infestação predial a menos que $1 \%$; diminuição do número de casos da doença em 50\%, em 2003, em relação aos registrados em 2002 e de $25 \%$ nos anos subsequentes; e redução da letalidade por febre hemorrágica de dengue a menos de $1 \%{ }^{3}$

No Brasil a gestão e execução das ações do PNCD são realizadas pelas secretarias municipais de saúde, com apoio dos estados e do Ministério da Saúde quando necessário, cabendo ao nível federal a maior parte do financiamento. Uma experiência de controle da dengue implementada com esse grau de descentralização, até o momento sem similar no mundo, ${ }^{4}$ requer para a sua efetividade na prevenção e controle a adequação das ações aos diferentes contextos loco-regionais; controle sobre as metodologias de intervenção, mantendo o caráter integral; realização de ações reconhecidas para produzir os efeitos esperados, de forma integrada, oportuna, em quantidade e qualidade. 5

A despeito do tempo decorrido e dos recursos investidos, o PNCD não tem alcançado os resultados esperados, 6,7 o que torna premente a realização de avaliações sobre o programa. Dois tipos iniciais de questões podem ser levantados: houve falha do programa em sua concepção e, como consequência, as atividades, mesmo sendo executadas, não alcançaram os efeitos desejáveis, ou houve falha na implementação do programa e com isso as atividades previstas não foram implantadas ou adequadamente desenvolvidas?

Uma das abordagens possíveis para observação desse problema é a realização de uma análise de coerência lógica do PNCD. Esse tipo de estudo, denominado análise de intervenção ou lógica do programa, enfoca aspectos relativos aos problemas de teoria, visando verificar a plausibilidade das relações causais entre os objetivos pretendidos para o programa e a adequação e suficiência das estratégias de ação propostas para alcançar tais resultados. Para vários autores esse tipo de análise é necessário para determinar se um programa teria ou não condição de ser avaliado, motivo pelo qual se referem a essa modalidade de estudo como estudo de avaliabilidade. 8,9

Diante dessas considerações, este estudo tem como objetivo avaliar a coerência lógica do PNCD quanto aos objetivos propostos e meios definidos para o alcance dos resultados, comparando-o com a Estratégia de Gestão Integrada - Dengue, da Organização Panamericana da Saúde (EGI-DengueOPAS), como modelo padrão proposto pela OPAS aos países das Américas. 10 O modelo EGI-DengueOPAS e o PNCD foram apresentados em seminário internacional por representantes do organismo internacional e do Ministério da Saúde (MS), respectivamente, e debatido com pesquisadores de centros de estudos e pesquisas nacionais sobre a adequação das estratégias para prevenção e controle da dengue.

\section{Métodos}

Estudo avaliativo do tipo análise lógica da intervenção, descritivo e qualitativo, no qual se utilizou como técnica de investigação a análise documental, considerada uma ferramenta adequada para subsidiar mapeamentos descritivos de políticas institucionais no sentido de indicar e sistematizar diferenças, contradições, confluências e insight sobre o discurso e as práticas programáticas. ${ }^{11}$ Três fontes de informação subsidiaram a análise: relatório de um painel de especialistas sobre dengue, 5 reunidos em Seminário Internacional realizado nos dias 18 e 19/07/2007 em Brasília para discussão de estratégias de avaliação do PNCD; revisão de estudos científicos publicados em periódicos ou não (teses e dissertações); e análise de documentos técnicos e de opinião.

Com base no relatório do painel de especialistas, 5 incluindo pesquisadores de instituições nacionais e internacionais das áreas epidemiológica, entomológica, de assistência e de educação e comunicação em saúde, profissionais e gestores do Ministério da Saúde e instâncias colegiadas do SUS, a análise de conteúdo procurou confrontar as convergências e divergências entre as intervenções e 
as lacunas de conhecimento existentes para o controle e prevenção da dengue.

Para a busca dos demais documentos, procedeuse revisão dos artigos e textos científicos realizada nas seguintes bases de dados: Scielo, Lilacs e Medline, utilizando como unitermos "dengue", "controle" e "avaliação", privilegiando-se os estudos avaliativos realizados no Brasil, no período de 1997 a 2007. Os documentos oficiais do e sobre o PNCD foram obtidos na página oficial do programa na web e com profissionais do Ministério da Saúde, incluindo: publicações normativas, relatórios técnicos, dados dos sistemas de informação e estudos previamente elaborados. Como critério de seleção para inclusão optou-se pelos estudos de avaliação da teoria, processo ou resultado do programa; textos de opinião de especialistas com análise sobre sua implantação ou efetividade; relatórios técnicos de avaliação da gestão, implementação das ações ou resultados do PNCD. No total foram identificados 79 textos, entre artigos científicos e de opinião, estudos avaliativos e documentos técnicos. Fez-se uma primeira leitura e classificação do material a partir dos resumos ou da íntegra do documento, sendo selecionados 27 textos para a análise da intervenção, compreendendo aqueles de interesse para apreciação do desenho, da implementação e dos possíveis resultados do programa. Após uma segunda leitura, esses foram sumarizados e descritos em uma tabela segundo os seus objetivos, metodologia e resultados, de acordo com os pressupostos e componentes do PNCD.

O PNCD foi descrito a partir dos documentos oficiais do programa ${ }^{3}$ e do modelo teórico-lógico elaborado por Pimenta Jr.12 Para análise da coerência, considerando os objetivos do programa e os meios empregados para alcançá-los, foi apreciado se o PNCD foi efetivo para atingir os objetivos gerais propostos - reduzir a infestação do mosquito, a incidência e letalidade da doença - e se os componentes do programa alcançaram os seus resultados específicos. Embora composto por dez componentes, optou-se neste estudo em apresentar a análise lógica do PNCD a partir de quatro deles, por reunirem maior número de estudos avaliativos, estarem presente em ambas as estratégias e as reflexões aparecem com maior ênfase no relatório do painel de especialistas. A EGI-Dengue-OPAS, tomada como modelo comparativo, foi analisada a partir do relatório do painel de especialistas ${ }^{5}$ e da estratégia elaborada para América Central e República Dominicana, modelo utilizado pela OPAS na elaboração das demais estratégias nacionais. ${ }^{2}$

\section{Resultados e Discussão}

Os documentos considerados para análise da intervenção encontram-se listados na Tabela 1 .

Os modelos de intervenção para prevenção e controle da dengue são descritos na Tabela 2 . Comparativos entre as estratégias e os quatro componentes selecionados pelo estudo são apresentados nas Tabelas 3 a 6.

\section{Comparativo entre as estratégias de intervenção para prevenção e controle da dengue: PNCD e EGI-Dengue-OPAS}

Os pressupostos que orientam as estratégias são convergentes, compreendendo a necessária adoção de um enfoque interprogramático, integrado e intersetorial, baseado em novas capacidades dos gestores e responsáveis e em práticas diferenciadas orientadas para o alcance de resultados, o que impõe o seu acompanhamento e avaliações sistemáticas. Os objetivos e metas são semelhantes, sendo que a meta brasileira para a letalidade para febre hemorrágica do dengue é mais ambiciosa que a proposta pela OPAS.

A lacuna em ambas refere-se à gestão do programa, com a não especificação da atuação esperada dos responsáveis pelos programas, de modo a promover localmente uma ação integral, integrada, intersetorial e participativa. A amplitude de ações previstas no PNCD requer habilidades diferenciadas na sua condução (técnica, política, de comunicação, de negociação), para que os gestores locais conduzam o programa de modo a garantir a sua implementação segundo a proposta vigente, ainda que sempre considerando as especificidades de cada contexto.

$\mathrm{Na}$ apreciação dos resultados das estratégias, observa-se que esses são em sua maioria negativos para o $\mathrm{PNCD}^{5,13,14}$ e positivos para a EGI-DengueOPAS. 5 Porém, cabe referir que enquanto os primeiros apresentam resultados de estudos avaliativos, para a estratégia EGI-Dengue os resultados referemse a levantamentos realizados pelo organismo internacional junto aos responsáveis pelo programa nos países, quando se discutem aspectos de fortalezas e debilidades no controle da dengue.

Quanto às estratégias estabelecidas para o alcance dos objetivos, as propostas trazem diferenças tanto na amplitude quanto no modo de organização dos componentes. Porém são coincidentes nos conteúdos dos componentes que estão presentes em ambas, como pode ser verificado nos quadros a seguir. 
Documentos incluídos na revisão para análise da intervenção. Brasil, 2008.

\begin{tabular}{|c|c|c|c|}
\hline Autor e ano da publicação & Local do estudo & $\begin{array}{c}\text { Tipo e Local de } \\
\text { publicação }\end{array}$ & $\begin{array}{l}\text { Componente } \\
\text { do PNCD }\end{array}$ \\
\hline Baglini et al.,2005.15 & São José do Rio Preto (SP) & $\begin{array}{l}\text { Artigo original } \\
\text { Cad Saúde Pública }\end{array}$ & 4 \\
\hline $\begin{array}{l}\text { Brasil. Ministério da Saúde. } \\
\text { Secretaria de Ciência, Tecnologia e } \\
\text { Insumos Estratégicos, 2006.16 }\end{array}$ & - & $\begin{array}{l}\text { Relatório técnico } \\
\text { MS, DECIT/CDTS }\end{array}$ & 1 e 2 \\
\hline $\begin{array}{l}\text { Brasil. Ministério da Saúde. } \\
\text { Secretaria de Vigilância em Saúde, } \\
2007.5\end{array}$ & - & $\begin{array}{l}\text { Relatório técnico } \\
\text { MS, SVS }\end{array}$ & $\begin{array}{l}\text { EGI-Dengue- } \\
\text { OPAS } \\
1,2,3 \text { e } 4\end{array}$ \\
\hline Brassolatti e Andrade, 2002.17 & Campinas (SP) & $\begin{array}{l}\text { Artigo original } \\
\text { Ciênc Saúde Coletiva }\end{array}$ & 4 \\
\hline Chiaravalloti et al., 2007.18 & São José do Rio Preto (SP) & $\begin{array}{l}\text { Artigo original } \\
\text { Cad Saúde Pública }\end{array}$ & 4 \\
\hline Chiaravalloti et al., 2006.19 & São José do Rio Preto (SP) & $\begin{array}{l}\text { Artigo original } \\
\text { Cad Saúde Pública }\end{array}$ & 4 \\
\hline Chiaravalloti et al., 1999.20 & São José do Rio Preto (SP) & Rev Soc Bras Med Trop & 2 e 4 \\
\hline Chiaravalloti et al., 1998.21 & São José do Rio Preto (SP) & $\begin{array}{l}\text { Artigo original } \\
\text { Cad Saúde Pública }\end{array}$ & 3 e 4 \\
\hline Chiaravalloti, 1997.22 & São José do Rio Preto (SP) & Cad Saúde Pública & 3 e 4 \\
\hline Chiaravalloti et al., 2002.23 & Catanduva (SP) & Cad Saúde Pública & 2 \\
\hline Corrêa e França, 2007.24 & Belo Horizonte (MG) & $\begin{array}{l}\text { Artigo original } \\
\text { Epidemiol Serv Saúde }\end{array}$ & 1 e 3 \\
\hline Donalísio et al., 2001.25 & Campinas (SP) & $\begin{array}{l}\text { Artigo originalRev Soc } \\
\text { Bras Med Trop }\end{array}$ & 3 e 4 \\
\hline Donalísio e Glasser, 2002.26 & - & $\begin{array}{l}\text { Artigo original } \\
\text { Rev Bras Epidemiol }\end{array}$ & 2 e 4 \\
\hline Forattini e Brito, 2003.27 & Potim (SP) & $\begin{array}{l}\text { Artigo original Rev } \\
\text { Saúde Pública }\end{array}$ & 2 e 4 \\
\hline Heintze et al., 2007.28 & - & $\begin{array}{l}\text { Revisão sistemática } \\
\text { Trans R Soc Trop Med } \\
\text { Hyg }\end{array}$ & 4 \\
\hline Kouri et al., 1986.29 & Cuba & $\begin{array}{l}\text { Bull Pan Am Health } \\
\text { Organ }\end{array}$ & 1 e 4 \\
\hline Lenzi et al., 2000.30 & Rio de Janeiro (RJ) & $\begin{array}{l}\text { Artigo original Cad } \\
\text { Saúde Pública }\end{array}$ & 4 \\
\hline Mello, 2006.13 & Rio de Janeiro (RJ) & $\begin{array}{l}\text { Dissertação de mestra- } \\
\text { do Rio de Janeiro: } \\
\text { ENSP/Fiocruz }\end{array}$ & 1,2 e 3 \\
\hline Oliveira et al., 2006.31 & Uberlândia (MG) & $\begin{array}{l}\text { Anais do III Encontro da } \\
\text { A N P P A S B r a síli a, } \\
\text { ANPPAS }\end{array}$ & 1 e 2 \\
\hline
\end{tabular}


Documentos incluídos na revisão para análise da intervenção. Brasil, 2008.

\begin{tabular}{|c|c|c|c|}
\hline Autor e ano da publicação & Local do estudo & $\begin{array}{c}\text { Tipo e Local de } \\
\text { publicação }\end{array}$ & $\begin{array}{l}\text { Componente } \\
\text { do PNCD }\end{array}$ \\
\hline Oliveira e Valla, 2001.32 & Rio de Janeiro (RJ) & $\begin{array}{l}\text { Artigo original } \\
\text { Cad Saúde Pública }\end{array}$ & 4 \\
\hline Penna, 2003.33 & - & $\begin{array}{l}\text { Artigo de opinião } \\
\text { Cad Saúde Pública }\end{array}$ & 1 e 2 \\
\hline Pimenta Jr, 2005.12 & Três municípios (MG) & $\begin{array}{l}\text { Dissertação } \\
\text { mestrado Rio de } \\
\text { Janeiro: ENSP/Fiocruz }\end{array}$ & $1,2,3$ e 4 \\
\hline Santos, 2003.14 & Macaparana, (PE) & $\begin{array}{lr}\text { Dissertação } & \text { de } \\
\text { mestradoRecife } & (P E): \\
\text { CPqAM/Fiocruz } & \end{array}$ & $1,2,3$ e 4 \\
\hline Tauil, 2002.4 & - & $\begin{array}{l}\text { Artigo de opinião } \\
\text { Cad Saúde Pública }\end{array}$ & 1 e 2 \\
\hline Teixeira et al., 2003.34 & Salvador (BA) & $\begin{array}{l}\text { Artigo original } \\
\text { Epidemiol Serv Saúde }\end{array}$ & 1 e 2 \\
\hline Teixeira et al., 2002.35 & Salvador (BA) & $\begin{array}{l}\text { Artigo original } \\
\text { Rev Bras Epidemiol }\end{array}$ & 1 e 2 \\
\hline Winch et al., 2002.36 & - & Am J Trop Med Hyg & 4 \\
\hline
\end{tabular}

PNCD=Programa Nacional de Controle da Dengue; $1=$ vigilância epidemiológica (integrada); $2=$ controle do vetor e entomologia; 3 = assistência aos pacientes; $4=$ ações integradas de educação em saúde, comunicação e mobilização social; EGI-Dengue-OPAS= Estratégia de Gestão Integrada - Dengue, da Organização Panamericana da Saúde. 
Comparativo entre as estratégias de intervenção para prevenção e controle da dengue: PNCD e EGI-Dengue-OPAS. Brasil, 2008.

\begin{tabular}{ll}
\hline PNCD & EGI-Dengue-OPAS \\
\hline
\end{tabular}

Pressupostos

Propósitos

Metas

Resultados da implementação das estratégias
- Necessidade de programa "permanente" pela impossibilidade de erradicação da dengue;

- Respeito aos princípios do Sistema Único de Saúde e inserção no contexto de descentralização do sistema de vigilância em saúde;

- Integração com as ações básicas de saúde

- Incorporar lições de experiências nacionais e internacionais de controle da dengue, implantando e intensificando ações visando um melhor enfrentamento do problema e redução do impacto da dengue no país

- Reduzir a menos de $1 \%$ a infestação predial em todos os municípios;

- Reduzir em 50\% o número de casos de 2003 em relação a 2002 e, nos anos seguintes, $25 \%$ a cada ano;

- Reduzir a letalidade por febre hemorrágica de dengue a menos de $1 \%$

- Avanços na incorporação de alguns componentes do programa na rotina do serviço;

- Dificuldade na implementação integral das ações previstas;

- Municípios reproduzem as ações tal como realizadas pelo governo federal, quando este assumia a execução centralizada do programa;

- Pouca capacidade local para implementação de um programa com a complexidade do PNCD, que requer diferentes competências (técnica, política, de comunicação, de negociação); 7,13,14

- Resultados positivos sobre a incidência da doença não podem ser computados à atuação do programa, podendo ser devido à ocorrência de epidemias anteriores e esgotamento de susceptíveis. Além disso, os índices de infestação vetorial não tiveram correlação direta com a incidência da dengue. 13
Nova geração de programas, orientado pela promoção da saúde e coordenação de ações entre governo, setor da saúde, setores econômicos e sociais, ONGs e organizações de apoio ao fortalecimento dos programas nacionais de prevenção e controle

Elaborar, implementar e consolidar uma estratégia de gestão integral e multisetorial para prevenção e controle da dengue

- Reduzir ocorrência de surtos;

- Diminuir magnitude e gravidade dos surtos;

- Reduzir em $50 \%$ a taxa de incidência em um período de cinco anos;

- Reduzir e manter a taxa de letalidade menor que $2 \%$ ao ano

- Maior coordenação intrassetorial (técnico como gerencial);

- Maior coordenação com outros setores, especialmente com os municípios;

- Melhor coordenação com grupos comunitários organizados na implementação de projetos locais de comunicação para mudança dos hábitos e condutas relacionados com a dengue;

- Aumento na capacidade de mobilização de recursos e maior ajuste do trabalho ao marco lógico, considerando custos das atividades e facilitação na negociação com investidores;

- Desenvolvimento de destrezas e habilidades na participação comunitária, educação e investigação antropológica;

- Maior capacidade de resposta e incorporação de novas ferramentas para vigilância epidemiológica, como o LIRAa e as novas armadilhas Mosquitrap.

PNCD=Programa Nacional de Controle da Dengue; EGI-Dengue-OPAS= Estratégia de Gestão Integrada - Dengue, da Organização Panamericana da Saúde, ONGs=organização não governamental; LIRAa=Levantamento Rápido de Índice Entomológico do Aedes aegypti. 
Comparativo entre o PNCD e a EGI-Dengue-OPAS quanto ao componente vigilância epidemiológica (integrada). Brasil, 2008.

\begin{tabular}{ll}
\hline PNCD & EGI-Dengue-OPAS \\
\hline
\end{tabular}

Objetivos

Resultados dos estudos
- Reduzir o número de casos e ocorrência de epidemias;

- Implementar atividades de controle oportunas;

- Produzir informes quinzenais;

- Monitorar a situação epidemiológica e entomológica com mapas municipais; SINAN como sistema de informação; FAD como fonte de informação vetorial;

- Executar levantamento rápido do índice larvário LIRAa;

- Capacitar técnicos para análise de dados;

- Manter rotina de monitoramento viral

\section{Resultados positivos:}

- Implantação de unidade ambulatorial de referência como estratégia para aprimorar a detecção e confirmação dos casos de dengue hemorrágica; 24 - Utilização de ovitrampa como armadilha eficiente para verificar a presença e os focos de ovos de Aedes aegypti nos locais armados e em diferentes períodos sazonais; 31

- Investigação de óbitos e coleta oportuna de sangue para o diagnóstico laboratorial; 13

- Melhoria da qualidade das informações e das análises realizadas e divulgadas, utilizando os sistemas disponíveis 12

\section{Resultados negativos:}

- Sistemas de informações atuando de forma passiva e pouco sensível para predizer surtos de dengue; ${ }^{5}$

- Pouca utilização das informações para orientar o combate ao vetor, ações educativas, saneamento e vigilância epidemiológica; subnotificação de casos e sistemas de informação pouco confiáveis; não rotinização da produção dos indicadores prioritários e do monitoramento epidemiológico e entomológico, conforme proposto pelo PNCD;12-14 - Subutilização dos dados do FAD e SINAN para orientar ações de combate ao vetor e vigilância epidemiológica; incoerência nos dados coletados na pesquisa e informados no DIAGDENGUE; situações importantes não qualificadas no sistema. 12
- Evitar ocorrência por infecções do vírus da dengue em áreas livres de circulação;

- Detectar precocemente as epidemias:

- Controlar as epidemias em curso;

- Reduzir a transmissão da doença nas áreas endêmicas - Reduzir a letalidade de febre hemorrágica e síndrome do choque

Fortalezas:

- Sistema de Vigilância Epidemiológica nos três níveis com cobertura nacional;

- Protocolos de Vigilância;

- Implementação de unidades sentinela para vigilância sorológica e virológica;

- Programa de formação contínua com profissionais e técnicos capacitados e comprometidos nos três níveis;

- Dengue como doença de notificação obrigatória com sistema de vigilância epidemiológica sensível para detecção precoce do aumento da transmissão e surtos;

- Normatização de responsabilidades;

- Vigilância comunitária em áreas de limitado acesso;

- Descentralização de técnicas diagnósticas;

- Equipes de apoio para situações de surtos e/ou emergências

Debilidades

- Recursos humanos insuficientes, com limitações no interior dos países e alta rotatividade; Inadequada e inoportuna notificação, e insuficiente classificação final dos casos;

- Insuficiente coordenação com os meios de comunicação e inexistência de comunicadores em saúde; - Gestão em diferentes níveis de desenvolvimento nos países com processos administrativos lentos e insuficientes:

- Dificuldade de confirmação laboratorial;

- Escassa experiência no diagnóstico e notificação de casos no interior dos países;

- Insuficiente seguimento e investigação de casos

PNCD=Programa Nacional de Controle da Dengue; EGI-Dengue-OPAS= Estratégia de Gestão Integrada - Dengue, da Organização Panamericana da Saúde; SINAN= Sistema de Informação de Agravos de Notificação; FAD=Sistema de Informação de Febre Amarela e Dengue; LIRAa=Levantamento Rápido de Índice Entomológico do Aedes aegypti. 
Comparativo entre o PNCD e a EGI-Dengue-OPAS quanto ao componente controle do vetor e entomologia. Brasil, 2008.

\begin{tabular}{|c|c|c|}
\hline & PNCD & EGI-Dengue-OPAS \\
\hline Objetivos & $\begin{array}{l}\text { - Implantar o FAD; } \\
\text { - Atualizar o número de imóveis; } \\
\text { - Unificar base geográfica; } \\
\text { - Viabilizar a correta utilização de equipamentos; } \\
\text { - Adequar o quantitativo de pessoal para combate ao } \\
\text { vetor; } \\
\text { - Viabilizar o combate ao vetor em portos e } \\
\text { fronteiras; } \\
\text { - Capacitar agentes e supervisores }\end{array}$ & $\begin{array}{l}\text { - Explicar a transmissão; } \\
\text { - Ajudar a avaliar as ações de prevenção e controle } \\
\text { do dengue; } \\
\text { - Desenhar novos métodos de avaliação, e controle }\end{array}$ \\
\hline $\begin{array}{l}\text { Resultados dos } \\
\text { estudos }\end{array}$ & $\begin{array}{l}\text { - Embora comprometa grande parte dos recursos } \\
\text { destinados ao programa (média de } 85 \% \text { ), não vem } \\
\text { sendo executado pelos municípios estudados } \\
\text { conforme o previsto; } 12 \\
\text { - Dificuldade no cumprimento das metas de } \\
\text { cobertura das ações pelos agentes de controle } \\
\text { vetorial e baixa efetividade do controle vetorial } \\
\text { centrado no trabalho dos guardas sanitários; } 33-35 \\
\text { - Divergências importantes quanto aos meios } \\
\text { empregados, o combate químico ao vetor, com } \\
\text { adoção de biocidas para eliminação das formas } \\
\text { imaturas ou adultas do Aedes aegypti.14 }\end{array}$ & $\begin{array}{l}\text { Problemas apontados na discussão do componente } \\
\text { indicando as fragilidades para o alcance dos } \\
\text { resultados esperados: } \\
\text { - Gestão: Complexidade no que se refere à aplicação } \\
\text { de meios antivetoriais físicos, biológicos e químicos } \\
\text { para controle de criadouros. Para a implementação } \\
\text { do controle químico nos cinco mil municípios no } \\
\text { Brasil, envolve multiplicar as atividades relativas à } \\
\text { contratação de pessoal, aquisição e manipulação de } \\
\text { produto, equipamento, transformando-se em algo } \\
\text { que requer uma atividade de alta gerência, aspecto } \\
\text { onde, infelizmente, se tem grandes limitações. }\end{array}$ \\
\hline $\begin{array}{l}\text { Resultados dos } \\
\text { estudos }\end{array}$ & $\begin{array}{l}\text { Poucos relatos sobre o uso de armadilhas, indicado } \\
\text { como efetivo para o controle do vetor, } 31 \text { bem como } \\
\text { do controle biológico como experiência de aplicação } \\
\text { em larga escala; } 33 \\
\text { - Desconhecimento quanto aos principais criadouros } \\
\text { do vetor existentes; } 27 \\
\text { - Na avaliação da efetividade de atividades visando à } \\
\text { redução da infestação, das duas atividades mais } \\
\text { realizadas para prevenção e controle de criadouros } \\
\text { do mosquito (casa-casa e arrastão), somente o casa- } \\
\text { casa mostrou-se correlacionado negativamente com } \\
\text { os níveis de infestação, sugerindo a baixa efetividade } \\
\text { dos arrastões. Neste caso a redução dos potenciais } \\
\text { criadouros do vetor não se refletiu no índice de } \\
\text { infestação entre as fases do estudo; } 20,23 \\
\text { - Dificuldade no controle do vetor parece indicar que } \\
\text { um programa centrado unicamente na redução de } \\
\text { criadouros potenciais dificilmente conseguirá evitar a } \\
\text { transmissão de dengue. Deve-se pensar em } \\
\text { estratégias complementares, como o uso de } \\
\text { armadilhas.31,35 }\end{array}$ & $\begin{array}{l}\text { Os esforços são, em geral, do tipo "emergenciais", } \\
\text { algo não planejado, mas realizado por pressão } \\
\text { social ou política ou estatal } \\
\text { - Técnico: estabelecer unidades operativas } \\
\text { entomológicas operativas, que não sejam unidades } \\
\text { especialistas, com mecanismos de controle de } \\
\text { qualidade. A entomologia necessita conhecer os } \\
\text { criadouros por área, e estar associada com as } \\
\text { estratégias de controle. Requer informação quanto } \\
\text { à associação de vetores e humanos, a suscetibilidade } \\
\text { aos inseticidas químicos e biológicos. Necessita } \\
\text { realizar monitoramento (insumos, processos e } \\
\text { produto), supervisão (qualidade e desempenho das } \\
\text { pessoas) e avaliação (levantamento dos resultados). } \\
\text { - Conhecimento científico: desconhecimento de } \\
\text { quantos mosquitos são necessários para que ocorra } \\
\text { uma epidemia. }\end{array}$ \\
\hline
\end{tabular}

PNCD=Programa Nacional de Controle da Dengue; EGI-Dengue-OPAS= Estratégia de Gestão Integrada - Dengue, da Organização Panamericana da Saúde; FAD=Sistema de Informação de Febre Amarela e Dengue. 
Comparativo entre o PNCD e a EGI-Dengue-OPAS quanto ao componente: Assistência aos pacientes. Brasil, 2008.

\begin{tabular}{lc}
\hline PNCD & EGI-Dengue-OPAS \\
\hline
\end{tabular}

Objetivos

Resultados dos estudos
Organização da rede assistencial; Promover a assistência adequada ao paciente

- A população, em sua maioria, não procura o serviço médico para o diagnóstico da dengue, 14 não considera a dengue uma doença grave e tem pouco conhecimento sobre a dengue hemorrágica;21,22,25 - Destacou-se um avanço na assistência ao paciente, explicado pela realização de capacitação dos profissionais de saúde para identificar clinicamente à dengue; 13

- Foi positiva a implantação de unidade ambulatorial de referência como estratégia para aprimorar a detecção e confirmação dos casos de dengue hemorrágica. 24
Diagnosticar e tratar oportunamente os pacientes de dengue

Fortalezas

- Protocolos definidos para as áreas de epidemiologia, assistência, controle do vetor e legislação;

- Notificação obrigatória regularmente para o nível federal;

- Capacitação continuada em todas as áreas;

- Rede de atenção hospitalar e ambulatorial

Debilidade

- Alta rotatividade de pessoal:

- Vínculos de trabalho

- Necessidade de capacitação contínua (aumento de custos)

PNCD=Programa Nacional de Controle da Dengue; EGI-Dengue-OPAS= Estratégia de Gestão Integrada - Dengue, da Organização Panamericana da Saúde.

Tabela 6

Comparativo entre o PNCD e a EGI-Dengue-OPAS quanto ao componente ações integradas de educação em saúde, comunicação e mobilização social. Brasil, 2008.

PNCD
Objetivos
- Educação em saúde: desenvolver ações educativas visando mudança de comportamento e
adoção de práticas para manutenção do ambiente domiciliar preservado da infestação por Aedes
aegypti;
- Comunicação social: divulgar e informar por meio de mídias as açães de educação em saúde e
mobilização social visando mudança de comportamento e hábitos da população, buscando evitar
a presença e reprodução do vetor nos domicílios.
Alto grau de conhecimento da população sobre o mosquito e a dengue clássica, em qualquer
grupo social; o conhecimento teve pouco impacto no comportamento e atitude das pessoas na
adoção das medidas de prevenção e controle da dengue; ações de educação em saúde,
comunicação e mobilização social referida como descontínuas e seguindo o modelo campanhista;
ações educativas realizadas sem planejamento organizado, sistematizado ou definido em função
dos fatores de risco identificados; mídia com papel preponderante na disseminação de
informação; identificação da necessidade de participação ativa da população, no planejamento e
execução das ações de educação em saúde e mobilização social; estratégias com envolvimento da
comunidade, aliada ao controle biológico ou químico do vetor, mostraram-se capazes reduzir os
índices larvários do Aedes aegypti, porém ainda se desconhece o efeito dessas estratégias na
transmissão da doença na população.4,12-15,17-19,21,22,25,30,32,35,36

EGI-Dengue-OPAS

PNCD=Programa Nacional de Controle da Dengue; EGI-Dengue-OPAS= Estratégia de Gestão Integrada - Dengue, da Organização Panamericana da Saúde. 
Considerando os achados dos estudos, apontam-se várias dificuldades para o desenvolvimento do programa pelos municípios. Embora com pouca referência sobre o que seria considerado como condições para gestão de um programa de controle da dengue, alguns aspectos problemáticos da implementação do PNCD são apontados: municípios reproduzem as ações de controle da dengue como realizada pelo governo federal quando este assumia de forma centralizada a execução do programa; integração intra e intersetorial seguem sendo um objetivo distante; pouca referência ao aspecto financeiro para gestão do programa. ${ }^{12-14}$

Os estudos avaliativos sobre o impacto do PNCD são ainda inexistentes. Os dois estudos encontrados de avaliação da efetividade referiam-se ao Programa de Erradicação do Aedes aegypti (PEAa), programa anterior ao atualmente vigente. Em estudo avaliando a institucionalização do PNCD, encontrou-se que nos dois municípios analisados houve redução da incidência de dengue nos últimos anos. Porém esses índices são reportados como provavelmente não confiáveis, pois os municípios não tiveram a cobertura domiciliar adequada do programa. 13

Comparativo entre o PNCD e a EGI-DengueOPAS quanto ao componente Vigilância Epidemiológica (integrada)

$\mathrm{Na}$ comparação do componente de vigilância epidemiológica na prevenção e controle da dengue entre as duas estratégias (Tabela 3) observa-se convergência nos objetivos. Em relação à EGIDengue-OPAS, os objetivos do componente são semelhantes aos objetivos gerais dessa intervenção, levando a supor que ao responsável pela área de vigilância epidemiológica na EGI corresponderia a responsabilidade pela gestão propriamente dita da estratégia. Quanto ao PNCD, pela extensão desse componente no desenho do programa brasileiro, é de se supor que recaia sobre o responsável pela implementação das suas ações, a coordenação do programa no âmbito local.

Os resultados apresentados para EGI-DengueOPAS foram sistematizados segundo as fortalezas, debilidades, oportunidades e ameaças; a Tabela 3 apresenta os dois primeiros aspectos. As fortalezas referidas à EGI-Dengue-OPAS no Brasil expressam avanços na implementação da vigilância epidemiológica para o controle da dengue, principalmente referente à normatização e educação continuada de profissionais. ${ }^{5}$ Quanto às debilidades apresentadas na avaliação da OPAS, essas coincidem com situações identificadas nos estudos avaliativos e opinião dos especialistas para o Brasil.

Como síntese da Tabela 3, os estudos avaliativos nacionais têm apontado os seguintes problemas para o desenvolvimento desse componente no âmbito local: baixa sensibilidade do sistema de vigilância epidemiológica para detecção precoce do aumento da transmissão e surtos, pouca e irregular produção de informações, insuficiente implementação de unidades sentinelas, pouco acompanhamento da atuação dos laboratórios. ${ }^{5,12-14}$ Chama atenção a pouca referência ao uso do sistema de informação especialmente construído para o monitoramento da implementação do programa PNCD pelos municípios, o DiagDengue. 12

Comparativo entre o PNCD e a EGI-DengueOPAS quanto ao componente Controle do vetor e Entomologia

A execução do componente controle do vetor e entomologia (Tabela 4) é dos mais complexos e onde se inserem várias controvérsias. Nesse ponto se enquadram os debates sobre os tipos de controle dos criadouros mais adequados, a efetividade das diferentes modalidades de intervenção, o papel das pessoas e dos setores públicos e as lacunas no conhecimento sobre questões-chave, tais como: qual o índice de infestação realmente necessário para evitar a transmissão da doença? Quais os principais criadouros do mosquito em cada contexto de atuação? Em que medida os indicadores utilizados estão fornecendo as informações necessárias e corretas sobre a circulação do mosquito? Algumas dessas questões têm sido levantadas por pesquisadores sobre o tema. 16,26

$\mathrm{Na}$ opinião do especialista da OPAS esse componente tem sido pouco contemplado nos últimos anos pelos novos gerentes, talvez por considerá-lo de difícil abordagem e que, sendo assim, seria melhor ignorá-lo, tratando apenas de reproduzir as orientações oriundas do nível federal. 5 Os resultados dos estudos e a opinião de especialistas no país indicam que as atividades nos municípios não têm sido implementadas conforme o previsto, 12 a cobertura das ações fica abaixo do programado, com dificuldade de alcance das metas e pouca participação de outros setores da saúde.21,22,33-35

A principal estratégia de controle vetorial segue sendo quase exclusivamente o uso de inseticidas contra o mosquito e os criadouros, com muito pouco sucesso nas atividades de controle físico e insuficiente discussão sobre controle biológico ou uso de armadilhas. 14,31,33 A resistência dos mosquitos aos inseticidas, bem como os problemas de saúde e ao 
meio ambiente decorrentes da utilização contínua e por vezes inadequada desses produtos foram também abordados. ${ }^{14}$ Outro aspecto a mencionar é o volume dos recursos do programa mobilizados para esse componente, que chegam a aproximadamente $85 \%$ dos valores executados. ${ }^{12}$ Como inovação teórica sobre o tema, foi referido no seminário 5 a utilização do conceito de vulnerabilidade das pessoas, para identificação dos potenciais riscos para epidemia, uma vez que o vetor isoladamente não é capaz de provocá-la nem de predizê-la.

Comparativo entre o PNCD e a EGI-DengueOPAS quanto ao componente Assistência aos pacientes

Embora não se possa prever se e quando um paciente com dengue clássica irá ou não se converter num caso de febre hemorrágica de dengue (FHD), é sabido que se essa evolução ocorrer tem-se que intervir o mais rapidamente possível para evitar que evolua para o choque por dengue, situação muito mais grave e que por suas complicações pode levar ao óbito. ${ }^{37}$ Para evitar esse risco, as atividades do componente 'Assistência aos Pacientes' (Tabela 5) enfatizam a capacitação dos profissionais e a elaboração dos planos de contingência. 3 O PNCD orienta aos executores a necessidade da organização e regulação da rede de assistência.

Um dos aspectos problemáticos da assistência aos pacientes referidos por especialistas nacionais no seminário internacional, e que repercute diretamente no indicador de letalidade da doença, é a classificação adotada para os casos de FHD. 5 A utilização dos parâmetros definidos pela Organização Mundial da Saúde (OMS), atualmente em uso no país, tem dificultado o encerramento de casos graves da doença que não atendem a todos os parâmetros, não sendo assim classificados como FHD. O que se observa no Brasil é uma grande notificação de casos, porém uma baixa ocorrência de dengue hemorrágica, o que pode resultar em uma letalidade alta por problemas na notificação.

Em um dos estudos analisados, assim como na análise apresentada pelo representante da OPAS para esse componente no seminário, cita-se a existência de unidade de referência para os pacientes de dengue como uma estratégia para melhorar a classificação dos casos, o manejo clínico dos pacientes e consequentemente reduzir as taxas de letalidade. 5,24

A prioridade a ser conferida ao componente para que se alcance o impacto esperado na redução da letalidade por dengue hemorrágico, um dos resultados finais previstos pelo programa, foi referida por vários participantes do seminário. A assistência aos pacientes, diferente da maioria dos demais, é de responsabilidade quase exclusiva do setor saúde e o desfecho dos casos de dengue que são assistidos pelos serviços é decorrência da qualidade da assistência prestada pelo sistema de saúde.

Comparativo entre o PNCD e a EGI-DengueOPAS quanto ao componente Ações integradas de educação em saúde, comunicação e mobilização social

A EGI-Dengue-OPAS trata o componente 'Ações Integradas de Educação em Saúde, Comunicação e Mobilização Social' (Tabela 6) com grande destaque e, no âmbito da estratégia, uma intervenção específica foi elaborada visando alcançar os objetivos: COMBI - Communication and Marketing Integrated for Behaviour Impact (Comunicação para Impactar Conduta) para o controle da Dengue. Esta é apresentada como um exercício de planejamento embasado em três elementos: estabelecimento de objetivos de conduta muito precisos; integração de ações de comunicação; e monitoramento contínuo. Segundo o especialista da OPAS, no momento não se encontra implementada no país nenhuma experiência de COMBI, com projetos pilotos em fase de avaliação. 5

Os resultados dos estudos referentes a esse componente no PNCD (Tabela 6) constata que enquanto o discurso normativo aponta para o desconhecimento e responsabilização das pessoas pelo problema, essas demonstram alto grau de conhecimento do problema, o que sugere que as informações sobre dengue estão bastante disseminadas, porém com pouco impacto sobre o comportamento quanto à adoção de práticas preventivas, $21,22,25$ e consideram o controle da dengue como responsabilidade do poder público, que não cumpre sua parte. 14,21,23 Os estudos, relacionando tais práticas com as experiências prévias das pessoas, demonstram: a visão negativa que têm, em geral, do serviço público; suas próprias prioridades, que não coincidem com as recomendações programáticas; as mensagens, às vezes equivocadas, que o serviço divulga; a proposição pelo serviço de medidas preventivas que ele mesmo não é capaz de executar; a falta de interesse em participar da prevenção; e a crença no caráter benigno e inevitável da doença. ${ }^{14,21,23,32,}$

Segundo a opinião de especialistas e a revisão bibliográfica, a informação repassada à população além de tardia é incompleta. Para se pensar na contribuição da população de forma efetiva e permanente se requer o repasse da melhor e mais recente 
informação possível; facilitar a comunicação com os responsáveis pelo controle vetorial; ter acesso aos meios disponíveis para controle do mosquito; participar ativamente no planejamento e execução das ações. 4,15,17,30,33 Intervenções multifacetadas com envolvimento dos serviços locais de saúde, profissionais capacitados pelos técnicos responsáveis pelo controle vetorial, participação do poder público e autoridades civis, comunidades atuando juntas para converter a informação em mudança da prática, encorajamento do papel ativo dos moradores na implementação das ações de prevenção e medidas de controle, parecem ser mais efetivas que intervenções simples, implementadas exclusivamente pelas secretarias de saúde e realizadas pelos agentes de controle de endemias. 17-19,28-30,32,36

Ao apreciarmos os estudos avaliativos e opinião dos especialistas sobre a implementação do PNCD como um todo e de alguns de seus componentes avaliados, cabe ressaltar que a inadequação verificada pode estar refletindo o pouco tempo decorrido entre a descentralização das ações de controle da dengue e a realização dos estudos analisados, tempo esse necessário para que os municípios implantassem as ações como previsto. Por outro lado, também, podem significar a pouca factibilidade do programa tal como formulado, considerando as condições político-institucionais e técnicas requeridas para a sua implementação, de difícil alcance pela maioria dos municípios. Além disso, a ação integrada não apenas localmente, mas envolvendo os demais âmbitos de gestão do SUS não pode deixar de ser considerada para o desenvolvimento de um programa da complexidade do PNCD.

\section{Considerações finais}

A partir da análise empreendida observou-se que é, ainda, reduzida a abrangência das avaliações sobre o PNCD. Os estudos consultados referem-se, principalmente, aos aspectos de processo e menos aos de efetividade do programa; a avaliação do programa em sua integralidade é objeto de poucos trabalhos e estes se referem apenas ao âmbito municipal de execução.

No que se refere à coerência do $\mathrm{PNCD}$, objeto da análise lógica da intervenção empreendida, identificou-se uma convergência nos pressupostos teóricos entre as estratégias comparadas, com similaridade dos objetivos e metas tanto gerais como dos componentes, bem como dos conteúdos previstos nas intervenções comuns a ambas. Convergem, também, nos aspectos mais problemáticos, referentes principalmente à estrutura e gestão dos programas.
A concordância verificada do ponto de vista normativo não se confirma na revisão dos estudos em três componentes: vigilância epidemiológica (integrada), controle do vetor e entomologia, e ações integradas de educação em saúde, comunicação e mobilização social. Quanto aos dois primeiros, aponta-se a atuação passiva da vigilância e o pouco uso das informações produzidas, bem como inadequação, inoportunidade e baixa qualidade das ações de controle vetorial e vigilância entomológica, com a indicação da necessidade de estudos e pesquisas básicas e epidemiológicas sobre o tema pela lacuna de conhecimentos existente, acarretando dificuldade para a atuação dos responsáveis pelas ações desse componente.

Com relação ao terceiro componente, os estudos indicam uma defasagem entre as mensagens e recomendações prescritas nas campanhas e material educativo, e a atitude da população diante da percepção do problema e dos meios necessários para agir sobre ele.

Quando se analisa a realização das ações e atividades propostas pelo PNCD pelas secretarias municipais de saúde, responsáveis pela execução das intervenções, embora seja possível identificar maior organização dessas ao incorporarem áreas técnicas responsáveis pela gestão do programa e disponibilidade de recursos específicos para sua execução, a implementação do programa mostrou-se invariavelmente inadequada.

Encontrou-se convergência entre a opinião dos especialistas e os achados dos estudos quanto à baixa efetividade das ações programáticas, indicada pelo não alcance dos resultados esperados. Porém, pouco se pode afirmar a respeito dos componentes que contribuíram mais ou menos com a situação observada, assim como sobre como os demais níveis de gestão estão atuando no apoio ou não à execução do programa. Ao se empreender estudos avaliativos para aferir a condição de implantação de intervenções é primordial observar todos os níveis de atuação, pois é previsto que o seu sucesso esteja na dependência da ação coordenada e oportuna de todos os âmbitos de gestão envolvidos.

As lacunas evidenciadas na análise comparativa entre o PNCD e o modelo EGI-Dengue-OPAS apontam para a baixa coerência e necessidade de atualização do modelo teórico-lógico do PNCD. Dada a baixa efetividade dos componentes analisados, considerando a ocorrência das epidemias e a incidência da dengue nos últimos vinte anos, o desenho do programa não tem se mostrado adequado (como modelo teórico-lógico) ou factível para responder satisfatoriamente ao problema. 
A implementação de ações mais pertinentes e serviços de melhor qualidade para alcance dos resultados esperados do PNCD no país, diferentemente do apresentado nos documentos oficiais - onde as ações de prevenção e controle são pouco específicas para os diferentes âmbitos de gestão e responsabilidade sobre o programa - está na dependência da definição de objetivos claros e metas factíveis para

\section{Referências}

1. World Health Organization. Dengue and dengue hemorrhagic fever. Fact sheet $N^{\circ} 117$. Geneve; 2009. [cited 2009 dez 14]. Available from: http://www.who.int/mediacentre/ factsheets/fs117/en/index.html.

2. San Martin JL, Brathwaite-Dick O. Integrated strategy for dengue prevention and control in the region of the Americas. Rev Panam Salud Pública. 2007; 21: 55-63.

3. Brasil. Ministério da Saúde. Fundação Nacional de Saúde (FUNASA). Programa Nacional de Controle da Dengue (PNCD). Brasília: FUNASA; 2002.

4. Tauil PL. Aspectos críticos do controle do dengue no Brasil. Cad Saúde Pública. 2002; 18: 867-71.

5. Brasil. Ministério da Saúde. Secretaria de Vigilância em Saúde. Relatório do Seminário Internacional de Avaliação do PNCD. Brasília, DF; 2007.

6. Barreto ML, Teixeira MG. Dengue no Brasil: situação epidemiológica e contribuições para uma agenda de pesquisa. Estud Av. 2008; 22: 53-72.

7. Pessanha JEM, Caiaffa WT, César CC, Proietti FA Avaliação do Plano Nacional de Controle da Dengue. Cad Saúde Pública. 2009 ; 25: 1637-41.

8. Champagne F, Brousselle A, Contandriopoulos A-P, Hartz Z. L'analyse stratégique. In: Brousselle A, Champagne F, Contandriopoulos A-P, Hartz Z, organizadores. Concepts et méthodes d'évaluation des interventions. Montréal: Les Presses de 1'Université de Montréal; 2009. p. 91-102.

9. Champagne F, Brousselle A, Contandriopoulos A-P., Hartz Z. L'analyse logique. In: Brousselle A., Champagne F. Contandriopoulos A-P, Hartz Z, organizadores. Concepts et méthodes d'évaluation des interventions. Montréal: Les Presses de 1'Université de Montréal; 2009. p. 103-12.

10. Organização Panamericana da Saúde. Dengue. Documento Técnico del 44. Consejo Directivo (CD 44/14). Washington: OPAS; 2003. [cited $2009 \mathrm{dez} 14$ ]. Available from: http://www.paho.org/spanish/gov/cd/cd44-14-s.pdf.

11. Natal S, Santos EM, Alves CKA, Felisberto E, Santos ES, Sousa A. A implementação do projeto estratégico de fortalecimento da capacidade técnica em monitoramento e avaliação das secretarias de saúde: avaliação formativa e autoavaliação. In: Hartz Z, Vieira-da-Silva LM, Felisberto E, organizadores. Meta-avaliação da atenção básica em saúde: teoria e prática. Rio de Janeiro: Editora Fiocruz; 2008. p. 341-67.

12. Pimenta Jr FG. Desenvolvimento e validação de um instrumento para avaliar o Programa Nacional de Controle da Dengue no âmbito municipal [dissertação]. Rio de Janeiro: Escola Nacional de Saúde Pública Sérgio Arouca da cada nível gerencial. Ressalta-se também a importância da atualização de conhecimentos sobre mecanismos mais eficazes de controle vetorial e modalidades de atuação de base comunitária, além da disponibilidade de recursos e autonomia em sua utilização a partir do nível de gestão responsável pela execução do programa.
Fundação Oswaldo Cruz; 2005.

13. Mello RCG. Modelo rápido de avaliação: estudo de caso da programação pactuada e integrada de epidemiologia e controle de doenças em municípios do estado do Rio de Janeiro [dissertação]. Rio de Janeiro: Escola Nacional de Saúde Pública Sérgio Arouca da Fundação Oswaldo Cruz; 2006.

14. Santos SL. Avaliação das ações de controle da dengue: aspectos críticos e percepção da população: Estudo de caso em um município do nordeste [dissertação]. Recife: Centro de Pesquisas Aggeu Magalhães da Fundação Oswaldo Cruz; 2003.

15. Baglini V, Favaro EA, Ferreira AC, Chiaravalloti Neto F, Mondini A, Dibo MR, Barbosa AAC, Ferraz AA, Cesarino MB. Atividades de controle do dengue na visão de seus agentes e da população atendida, São José do Rio Preto, São Paulo, Brasil. Cad Saúde Pública. 2005; 21: 1142-52.

16. Brasil. Ministério da Saúde. Secretaria de Ciência, Tecnologia e Insumos Estratégicos. Relatório da Oficina de Prioridades em Doenças Negligenciadas. Brasília, DF; 2006.

17. Brassolatti RC, Andrade CFS. Avaliação de uma intervenção educativa na prevenção da dengue. Ciênc Saúde Coletiva. 2002; 7: 243-51

18. Chiaravalloti Neto F, Baglini V, Cesarino MB, Favaro EA, Mondini A, Ferreira AC, Dibo MR, Barbosa AAC, Ferraz AA. O programa de controle do dengue em São José do Rio Preto, São Paulo, Brasil: dificuldades para a atuação dos agentes e adesão da população. Cad Saúde Pública. 2007; 23: $1656-64$.

19. Chiaravalloti Neto F, Barbosa AAC, Cesarino MB, Favaro EA, Mondini A, Ferraz AA, Dibo MR, Vicentini ME. Controle do dengue em uma área urbana do Brasil: avaliação do impacto do Programa Saúde da Família com relação ao programa tradicional de controle. Cad Saúde Pública. 2006; 22: 987-97.

20. Chiaravalloti Neto F, Costa AIP, Moura MSA, Soares MRD, Pereira FC, Battigaglia M, Aragão FJOS. Avaliação de ações municipais de combate a vetores da dengue na região de São José do Rio Preto, São Paulo, 1989 a 1995. Rev Soc Bras Med Trop. 1999; 32: 357-62.

21. Chiaravalloti Neto FC, Moraes MS, Fernandes MA. Avaliação dos resultados de atividades de incentivo à participação da comunidade no controle da dengue em um bairro periférico do Município de São José do Rio Preto, São Paulo, e da relação entre conhecimentos e práticas desta população. Cad Saúde Pública. 1998; 14 (Supl 2): $101-9$. 
22. Chiaravalloti Neto FC. Conhecimentos da população sobre dengue, seus vetores e medidas de controle em São José do Rio Preto, São Paulo. Cad Saúde Pública. 1997; 13: $447-$ 53.

23. Chiaravalloti VB, Moraes MS, Chiaravalloti Neto F, Conversani DT, Fiorin AM, Barbosa AAC. Avaliação sobre a adesão às práticas preventivas do dengue: o caso de Catanduva, São Paulo, Brasil. Cad Saúde Pública. 2002; 18: 1321-9.

24. Corrêa PRL, França E. Dengue hemorrágica em unidade de referência como indicador de sub-registro de casos no município de Belo Horizonte, Estado de Minas Gerais, Brasil, 1998. Epidemiol Serv Saúde. 2007; 16: 175-84.

25. Donalísio MR, Alves JCP, Visockas A. Inquérito sobre conhecimentos e atitudes da população sobre a transmissão do dengue - região de Campinas, São Paulo, Brasil - 1998 Rev Soc Bras Med Trop. 2001; 34: 197-201

26. Donalísio MR, Glasser CM. Vigilância entomológica e controle de vetores do dengue. Rev Bras Epidemiol. 2002; 5: $259-70$

27. Forattini OP, Brito M. Reservatórios domiciliares de água e controle do Aedes aegypti. Rev Saúde Pública. 2003; 37: 676-7

28. Heintze C, Garrido M, Velasco KA. What do communitybased dengue control programmes achieve? A systematic review of published evaluations. Trans R Soc Trop Med Hyg. 2007; 101: 317-25.

29. Kouri GP, Guzman MG, Bravo JR. Dengue hemorrágica en Cuba. Crônica de una epidemia. Bull Pan Am Health Organ. 1986; 100: 322-29.

30. Lenzi MF, Camilo-Coura L, Grault CE, Val MB. Estudo do dengue em área urbana favelizada do Rio de Janeiro: considerações iniciais. Cad Saúde Pública. 2000; 16: 851-6.
31. Oliveira JC, Lima SC, Lemos JC, Casagrande B, Borges EA, Silva DB, Ferrete JA, Rezende K. A utilização da ovitrampa como prevenção do Aedes e controle do Dengue no Distrito de Martinésia, Uberlândia (MG). In: III Encontro da Associação Nacional de Pós-Graduação e Pesquisa em Ambiente e Sociedade; 2006 Mai 23-26; Anais do III Encontro da ANPPAS. Brasília: ANPPAS. 16 p. Disponível em: http://www.anppas.org.br/encontro_anual/encontro3/ arquivos/TA275-09032006-175529.PDF

32. Oliveira RM, Valla VV. As condições e as experiências de vida de grupos populares no Rio de Janeiro: repensando a mobilização popular no controle do dengue. Cad Saúde Pública. 2001; 17 (Supl): 77-88.

33. Penna MLF. Um desafio para a saúde pública brasileira: o controle do dengue. Cad Saúde Pública. 2003; 19: 305-9.

34. Teixeira MG, Barreto ML, Costa MCN, Ferreira LDA, Vasconcelos P. Dinâmica de circulação do vírus da dengue em uma área metropolitana do Brasil. Epidemiol Serv Saúde. 2003; 12: 87-97.

35. Teixeira MG, Barreto ML, Costa MCN, Ferreira LDA, Vasconcelos PFC. Avaliação de impacto de ações de combate ao Aedes aegypti na cidade de Salvador, Bahia. Rev Bras Epidemiol. 2002; 5: 108-15.

36. Winch PJ, Leontsini E, Rigau-Pérez JG, Ruiz-Pérez M, Clark GG, Gubler DJ. Community-based dengue prevention programs in Puerto Rico: impact on knowledge, behavior, and residential mosquito infestation. Am J Trop Med Hyg. 2002; 67: 363-70.

37. Martinez E. La prevención de la mortalidad por dengue: un espacio e um reto para la atención primaria de salud. Rev. Panam Salud Publica. 2006, 20: 60-74.

Recebido em 17 de março de 2010

Versão final apresentada em 2 de julho de 2010

Aprovado em 19 de julho de 2010 